\title{
Conjugate properties of magnetospheric Pc5 waves: AntarcticaGreenland comparison
}

Pilipenko, Vyacheslav A.; Martines-Bedenko, Valery A.; Coyle, Shane; Fedorov, Evgeny N.; Hartinger, Michael D.; Engebretson, Mark J.; Edwards, Thom R.

Published in:

Journal of Geophysical Research: Space Physics

Link to article, DOI:

10.1029/2020JA028048

Publication date:

2021

Document Version

Publisher's PDF, also known as Version of record

Link back to DTU Orbit

Citation $(A P A)$ :

Pilipenko, V. A., Martines-Bedenko, V. A., Coyle, S., Fedorov, E. N., Hartinger, M. D., Engebretson, M. J., \& Edwards, T. R. (2021). Conjugate properties of magnetospheric Pc5 waves: AntarcticaGreenland comparison. Journal of Geophysical Research: Space Physics, 126(2), [e2020JA028048].

https://doi.org/10.1029/2020JA028048

\section{General rights}

Copyright and moral rights for the publications made accessible in the public portal are retained by the authors and/or other copyright owners and it is a condition of accessing publications that users recognise and abide by the legal requirements associated with these rights.

- Users may download and print one copy of any publication from the public portal for the purpose of private study or research.

- You may not further distribute the material or use it for any profit-making activity or commercial gain

- You may freely distribute the URL identifying the publication in the public portal 


\section{JGR Space Physics}

\section{RESEARCH ARTICLE \\ 10.1029/2020JA028048 \\ Conjugate Properties of Magnetospheric Pc5 Waves: Antarctica-Greenland Comparison}

\section{Special Section:}

Probing the Magnetosphere through Magnetoseismology and Ultra-Low-Frequency Waves

Key Points:

- Pc5 waves recorded at conjugate magnetometers in Greenland and Antarctica are analyzed from viewpoint of voltage/current generator dichotomy

- During winter/summer events spectral Pc5 power is about the same, though the contrast between ionospheric conductances is $5-10$ times

- The conjugacy properties of resonant Pc5 waves in Greenland and Antarctica correspond well to the current generator regime

Correspondence to:

V. A. Pilipenko,

space.soliton@gmail.com

Citation:

Pilipenko, V. A., Martines-Bedenko, V. A., Coyle, S., Fedorov, E. N., Hartinger, M. D., Engebretson, M. J., \& Edwards, T. R. (2021). Conjugate properties of magnetospheric Pc5 waves: AntarcticaGreenland comparison. Journal of Geophysical Research: Space Physics, 126, e2020JA028048. https://doi. org/10.1029/2020JA028048

Received 26 MAR 2020 Accepted 16 JAN 2021

\section{Introduction: Current/Voltage Dichotomy of ULF Waves}

\author{
Vyacheslav A. Pilipenko ${ }^{1,2} \mathbb{D}$, Valery A. Martines-Bedenko ${ }^{2}$, Shane Coyle $^{3} \mathbb{C}$, \\ Evgeny N. Fedorov ${ }^{2}$, Michael D. Hartinger ${ }^{3,4}$ (D) Mark J. Engebretson ${ }^{5} \mathbb{D}$, and Thom R. Edwards ${ }^{6}$ \\ ${ }^{1}$ Space Research Institute, Moscow, Russia, ${ }^{2}$ Institute of Physics of the Earth, Moscow, Russia, ${ }^{3}$ Bradley Department \\ of Electrical and Computer Engineering, Virginia Tech, Blacksburg, Virginia, USA, ${ }^{4}$ Space Science Institute, \\ Colorado, USA, ${ }^{5}$ Augsburg University, Minneapolis, Minnesota, USA, ${ }^{6}$ DTU Space, Technical University of Denmark, \\ Copenhagen, Denmark
}

\begin{abstract}
An electric circuit analogy for magnetosphere-ionosphere current systems has two extremes for their driver: a current generator and voltage generator. In the case of a magnetospheric current generator the ground magnetic effect must be nearly the same under low-conductivity or highconductivity ionospheres, whereas in the case of a voltage generator the magnetic response must be larger under a high-conductivity ionosphere. Theoretical consideration of the field-aligned current generation by a magnetospheric driver within the framework of the magnetosphere "plasma box" model with asymmetric ionospheres showed that excitation of resonant Alfvenic oscillations should correspond rather to a current generator regime. The asymmetry of Pc5 waves (frequencies about several $\mathrm{mHz}$ ) recorded at conjugate magnetometers in Greenland and Antarctica has been analyzed from the viewpoint of the voltage/current generator dichotomy. We have selected Pc5 events under contrasting ionospheric conditions, during summer and winter. For resonant frequencies, we have plotted the latitudinal profiles of spectral power and compared the amplitudes of spatial maxima in opposite hemispheres. Along the same profile, latitudinal dependences of the ionospheric height-integrated conductivities have been reconstructed with the use of the OVATION-prime (OP) and solar illumination models. During both winter and summer events, the amplitudes of the spectral power peak in Greenland and Antarctica have been found to be about the same, though the OP model predicts a contrast between ionospheric conductivities of 5-10 times. Thus, the interhemispheric properties of Pc5 waves correspond to the current generator regime, as predicted by the magnetospheric plasma box model.
\end{abstract}

Observational and modeling studies have shown the interhemispheric symmetry/asymmetry in geomagnetic field disturbances and its effects on magnetosphere-ionosphere coupling that are manifested in largescale current systems, auroral forms, waves, plasma upflow/outflow, particle precipitation, high-latitude convection, etc. (Zesta et al., 2016). Interhemispheric differences and their effects on magnetosphere-ionosphere coupling are to be incorporated in observations and modeling/simulations. Large-scale electromagnetic disturbances are transported from the equatorial plane of the magnetosphere towards the high-latitude ionosphere via field-aligned currents (FACs). When considering drivers of the magnetosphere-ionosphere current system it is physically intuitive to classify them as either a fixed-current generator, or fixed-voltage generator (Lysak, 1985; Sibeck et al., 1996). These extreme cases correspond to contrasting ratios between the generator internal resistance and the load on the circuit (Lysak, 1990). Magnetosphere-ionosphere nonsteady current systems with typical time scales in the UltraLow Frequency (ULF) band (time scales 1-10 min) can be also described using this electrical circuit analogy, with the ionosphere functioning as a load and a process in the magnetosphere functioning as a generator (Lam \& Rodger, 2004). If the external process driving the ionospheric electric field behaves as a voltage generator, then the electric field remains constant while the ionospheric current and magnetic response vary upon a change of the ionospheric conductivity. In contrast, if the external process behaves as a current generator, the ionospheric current and magnetic field intensities remain fixed while electric field may vary upon a change of the ionospheric conductivity.

There are several possible ways to examine the dependence of ground magnetic response to a magnetospheric driver on the ionospheric conductance. The first one is to study the diurnal variations of ground
(C) 2021. American Geophysical Union. All Rights Reserved. 
magnetic disturbance amplitude at a selected site. However, the intensity of a magnetospheric driver may not be the same throughout a day. The second way is to determine the seasonal variation of the magnetic disturbance amplitude. Upon examining the seasonal variations, for a magnetospheric FAC generator the local ionosphere plays the role of a load resistance, whereas the Alfven wave resistance of the magnetosphere and the conjugate ionosphere play the role of a source resistance (Pilipenko et al., 2019). The influence of several unrelated parameters makes an unambiguous interpretation of seasonal variations difficult. Finally, the current/voltage paradigm can be tested with simultaneous observations at conjugate sites under asymmetric ionospheres.

This last approach was applied to find the driver characteristic of travelling convection vortices (Kim et al., 2015) and storm sudden commencements (Pilipenko et al., 2020). The examination of conjugacy properties may be an effective tool to comprehend the physics of magnetospheric ULF waves (Obana et al., 2005). ULF waves in the Pc5 band (periods about 3-10 min) are observed by ground magnetometers on the morning flank at auroral latitudes as quasi-monochromatic long-lasting (up to several hours) pulsations. The occurrence of these waves is due to the response of a natural Alfven resonator formed by magnetospheric field lines between conjugate ionospheres to an external driving. The toroidal (azimuthally largescale) Pc5 waves in the magnetospheric resonator are excited by shear-flow instability at the magnetopause driven by fast magnetosheath plasma flow around the magnetosphere or solar wind pressure pulses. Waves excited by these sources differ by their waveforms: extra-magnetospheric pulses excite transient oscillations, whereas flow-driven waves are long-lasting (as a flag swings in the wind). However, this distinction is not very strict, pressure pulses in the high-speed solar wind increase the growth rate of the shear-flow instability (Mishin, 1993). Pc5 waves are the most powerful electromagnetic wave phenomenon in the near-Earth space, and they can modulate fluxes of trapped and precipitated energetic particles, modify the ionospheric plasma, and pump energy into the radiation belt electrons (Menk \& Waters, 2013).

An important aspect of the magnetospheric disturbance interaction with the ionosphere which should be taken into account when considering the current/voltage dichotomy is that nonsteady FACs interact with the ionosphere in a different way in regimes of forced driving or resonant excitation of field line oscillations. Pilipenko et al. (2019) considered a simple "plasma box" model of the magnetospheric field line Alfven resonator with asymmetric conjugate ionospheres which is driven by an external current, and compared the ground magnetic responses in both hemispheres. The theoretical predictions of this model fit rather well the reported conjugacy properties of magnetic bays and travelling convection vortices. The predictions of this model for ULF Pc5 waves are summarized in section 3. We analyze the results of observations of Pc5 waves at conjugate magnetometer arrays in Greenland and Antarctica from the viewpoint of the voltage/ current generator dichotomy.

\section{The Model of the Magnetospheric Field Line Resonator With Asymmetric Ionospheres}

The ionospheric and ground response to magnetospheric disturbances is primarily caused by nonsteady FACs transported by transient or standing Alfven waves. MHD disturbances in a "magnetospheric box" with a straight magnetic field $\mathbf{B}_{0}$ turn out to be, though simple, a qualitatively adequate model of magnetospheric ULF waves (Southwood, 1974). A sketch of the magnetospheric box model is given in Figure 1. The coordinate $x$ corresponds to the Earthward radial direction in the magnetosphere (N-S direction on the ground), the $z$ axis is along $\mathbf{B}_{0}$, and the $y$ axis corresponds to the azimuthal direction in the magnetosphere (E-W direction on the ground). Field lines with length $2 L$ are terminated by conjugate Southern (S) and Northern (N) infinitely thin ionospheres with height-integrated Pedersen/Hall conductances $\Sigma_{P, H}^{(S)}$ and $\Sigma_{P, H}^{(N)}$ . The fundamental eigenfrequency of an Alfven resonator with a homogeneous distribution of the Alfven velocity $V_{A}$ along a field line is $\Omega_{A}=\pi V_{A} / 2 L$. The oscillations are driven by an external transverse current localized at the magnetospheric equator $(z=0)$. The efficiency of the Alfvenic disturbance reflection from the ionosphere is determined by the contrast between the ionospheric Pedersen conductance $\Sigma_{P}$ and magnetospheric Alfven wave conductance $\Sigma_{A}=\left(\mu_{0} V_{A}\right)^{-1}$, where $\mu_{0}$ is the magnetic constant. 

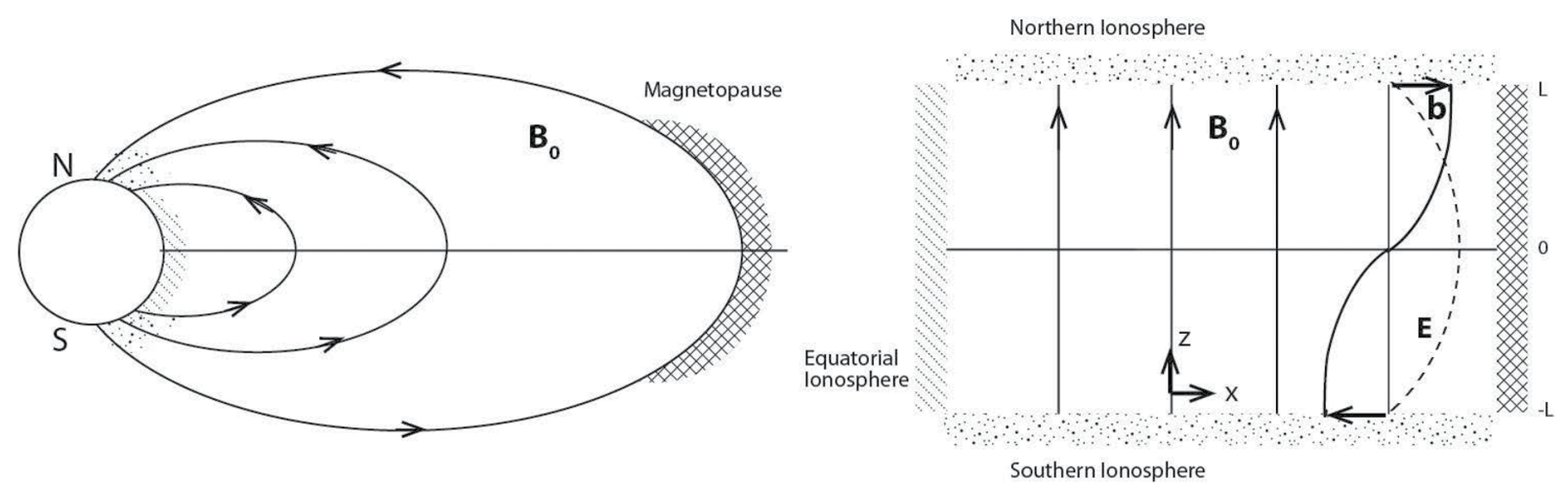

Figure 1. A sketch of the "magnetospheric box" model: homogeneous plasma with constant Alfven velocity $V_{A}$ immersed in a straight magnetic field $B_{0}$. The field lines with length $2 L$ are terminated by the conjugate Southern $(\mathrm{S})$ and Northern $(\mathrm{N})$ ionospheres with conductances $\Sigma_{P}^{(S)}$ and $\Sigma_{P}^{(N)}$. The field line oscillations are driven by an external transverse current $j_{x}^{(d)}$.

\subsection{Ratio Between Magnetic Responses in Conjugate Sites}

The theoretical modeling of FAC excitation by a driver in the equatorial plane of the magnetosphere (Pilipenko et al., 2019) gave the following estimates of the ratio between ground magnetic responses in geomagnetically conjugate points. In the case of quasi-DC forced driving, when the characteristic frequency of an external driver is much less than the field line eigenfrequency, $\omega \ll \Omega_{A}$, the ratio between the ground magnetic disturbances at conjugate points is to be as follows

$$
\frac{B_{g}^{(N)}}{B_{g}^{(S)}}=\frac{\Sigma_{H}^{(N)}}{\Sigma_{H}^{(S)}} \frac{\Sigma_{A}^{(S)}}{\Sigma_{A}^{(N)}}
$$

Thus, the ground magnetic response is to be proportional to the local ionospheric Hall conductance, because the Alfven wave conductance $\Sigma_{A}$ is determined by the magnetospheric plasma distribution, but not by the properties of the ionosphere.

In the case of resonant driving, when the frequency of the oscillatory driver matches the local field line frequency of a magnetic shell, $\omega \rightarrow \Omega_{A}$, the ratio of ground magnetic responses to Alfvenic toroidal-type (azimuthally large-scale) waves in conjugate points is as follows:

$$
\frac{B_{g}^{(N)}}{B_{g}^{(S)}} \simeq \frac{T^{(N)}}{T^{(S)}}, \quad T=\frac{\Sigma_{H}}{\Sigma_{P}}
$$

The ratio between the local Hall and Pedersen conductances remains nearly the same throughout a day, $T \simeq 1-2$, therefore the ratio of ground magnetic responses in conjugate points should not depend much on variation of the ionospheric conductance. This behavior corresponds to the current generator regime.

Formally, in the model considered here the magnetic field and plasma are assumed to be laterally homogeneous. In reality, an external MHD disturbance (surface or fast compressional modes) can excite FAC only thanks to mode coupling in an inhomogeneous system as will be considered in the next section.

\subsection{Interhemispheric Properties of the Resonant Quarter-Wave Mode}

The Alfven wave reflection coefficient $R=\left(\bar{\Sigma}_{P}-1\right) /\left(\bar{\Sigma}_{P}+1\right)$ depends not on just ionospheric conductance, but on the ratio between the Pedersen conductance and magnetospheric wave conductance $\bar{\Sigma}_{P}^{(S, N)}=\Sigma_{P}^{(S, N)} / \Sigma_{A}$. Depending on the ratio between $\bar{\Sigma}_{P}$ at conjugate points, two types of Alfven resonance modes are possible: (1) The half-wave mode when the reflection coefficients are either both positive $R^{(S)}>0$, $R^{(N)}>0$ (Sun-illuminated ionospheres), or both negative, $R^{(S)}<0, R^{(N)}<0$ (dark ionospheres). (2) The quarter-wave mode, when $R^{(S)} R^{(N)}<0$, that is, when one ionosphere is high-conductive, whereas the other one is low-conductive. The real part of the fundamental eigenfrequency of this wave mode is two times less than 
that of a half-wave mode, $\Omega_{A}=\pi V_{A} / 4 L$. Low-damping oscillations are possible when $\bar{\Sigma}_{P} \gg 1$ or $\bar{\Sigma}_{P} \ll 1$. The question arises whether the relationship between Northern and Southern hemispheres would be the same for resonant half-wave and quarter-wave modes? In the case of quarter-wave resonance the ratio between magnetic responses on the ground in conjugate sites can be derived from general relationships of the "plasma box" model (Pilipenko et al., 2019)

$$
\frac{B_{N}^{(g)}}{B_{S}^{(g)}} \simeq-\frac{\bar{\Sigma}_{H}^{(N)}}{\bar{\Sigma}_{H}^{(S)}} \frac{1-i \bar{\Sigma}_{P}^{(S)}}{1-i \bar{\Sigma}_{P}^{(N)}}
$$

Let us consider low-damping magnetospheric field line oscillations between the high-conductive Northern ionosphere and low-conductive Southern ionosphere, that is $\bar{\Sigma}_{P, H}^{(N)} \gg 1$ and $\bar{\Sigma}_{P, H}^{(S)} \ll 1$. In this case (3) reduces to

$$
\frac{B_{N}^{(g)}}{B_{S}^{(g)}} \simeq i \frac{T^{(N)}}{\bar{\Sigma}_{H}^{(S)}}
$$

Thus, beneath the high-conductive ionosphere the ground magnetic response is to be much larger than that beneath a low-conductive ionosphere, $\left|B_{N}^{(g)} / B_{S}^{(g)}\right| \gg 1$.

\subsection{Spatial Structure of the Resonant ULF field}

Periodic large-scale MHD disturbances from remote parts of the magnetosphere propagate inside the magnetosphere and, through a mode transformation, excite standing toroidal Alfven oscillations of the Earth's magnetic field lines. The mode conversion is most effective in the vicinity of the resonant shell where the local eigenfrequency $f_{A}(x)$ of Alfven field line oscillations coincides with the frequency $f$ of an external source (Chen \& Hasegawa, 1974). The mathematical description of the spatial structure of the coupled MHD modes can be expressed in the form of the Frobenius expansion in the vicinity of a resonant field line (Kivelson \& Southwood, 1986; Krylov et al., 1980). The expansion of the azimuthal magnetic component $B_{y}(x, f)$ has a singularity at a resonant shell $\left(x \rightarrow x_{A}(f)\right)$. At the same time, the radial $B_{x}(x, f)$ component has just a weak logarithmic singularity near the resonance, so its resonant behavior would hardly be noticeable. The spectral MHD theory Krylov et al. (1979) states that the frequency and field-aligned structure of Alfvenic-type oscillations excited by an external MHD disturbance are described by ordinary differential 1D equations, identical to equations for the uncoupled Alfven modes in a homogeneous plasma.

Upon transmission through the ionosphere, the horizontal spatial structure of ULF waves is distorted. This distortion can be analytically described for the Alfven wave with a Lorentz-type spatial structure transmitting through the "thin" ionospheric layer above an infinitely conductive ground (Alperovich \& Fedorov, 2007; Hughes \& Southwood, 1976). On the ground oscillations keep the same spatial form with account for two factors: (a) the $\pi / 2$ rotation of the wave polarization ellipse: $B_{y}^{(m)} \rightarrow B_{x}^{(g)}$ (north-south component at the ground), $B_{x}^{(m)} \rightarrow B_{y}^{(g)}$ (east-west component at the ground); (b) the widening of the resonance peak $\delta_{g}$, as observed at the ground, as compared with that above the ionosphere, $\delta_{g}=\delta_{m}+h$.

The leading term which describes amplitude of the N-S ULF wave component at the ground $B_{g} \equiv B_{x}$ in the vicinity of a resonant magnetic shell can be written as (Pilipenko et al., 2016):

$$
\left|B_{g}(x, f)\right|=B_{o}(f) \frac{\delta_{m}}{\sqrt{\left[x-x_{A}(f)\right]^{2}+\delta_{g}^{2}}}
$$

According to (5), the latitudinal structure of the ULF field can be qualitatively represented as the combination of a "source" spectrum and a magnetospheric Alfvenic resonance response. The "source" part is related to a disturbance transported by a large-scale fast compressional wave and has a weak dependence on the $x$ coordinate. The resonant magnetospheric response related to the Alfven wave excitation is strongly localized and it causes rapid enhancement of amplitude when a resonant shell is approached. The width $\delta_{g}$ of the resonant response is determined by the dominant damping mechanism (Yumoto et al., 1995). One of 


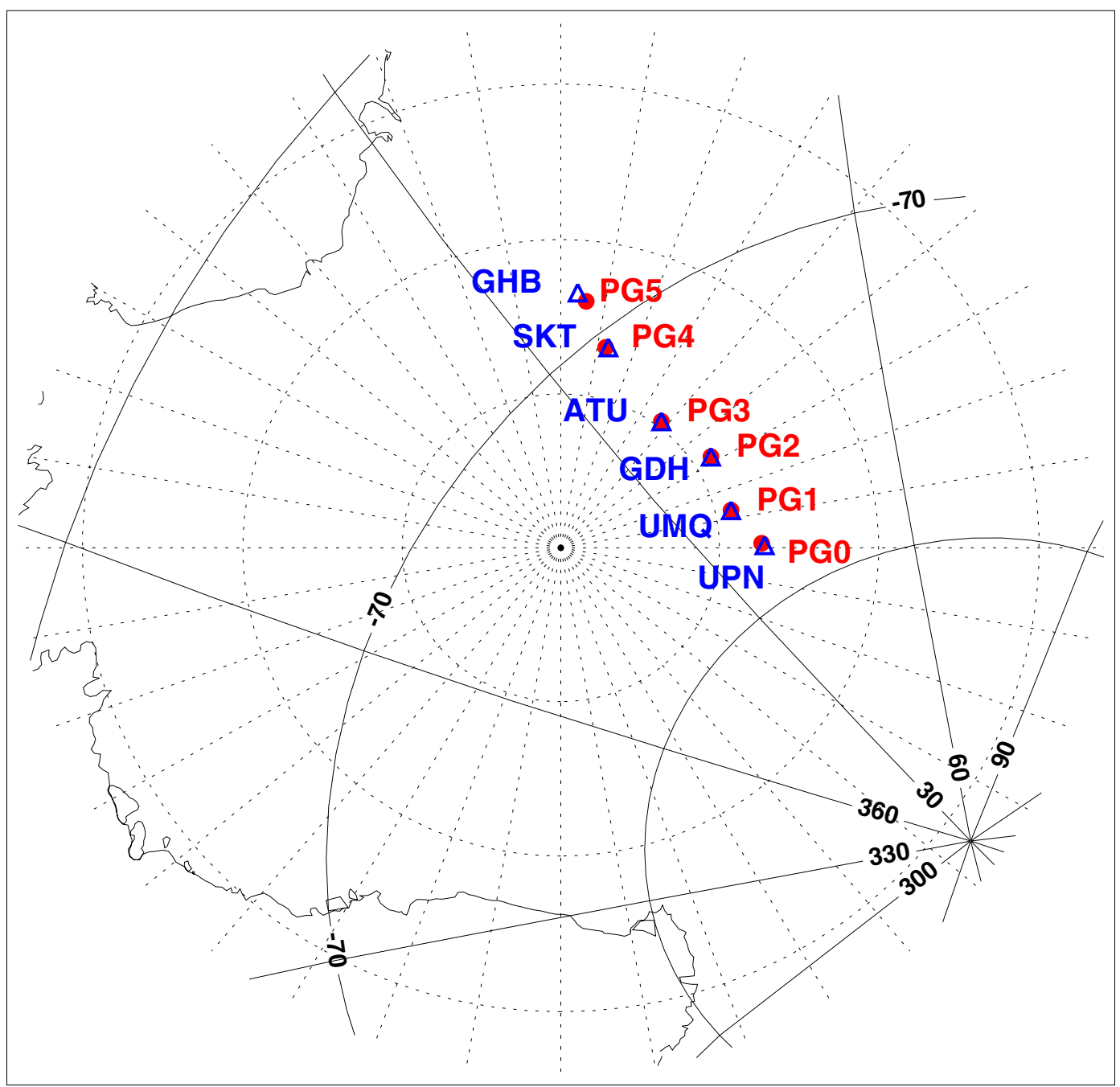

Figure 2. A map of the Antarctic magnetometer stations (red dots) used in this study. The geomagnetic projection of Greenland stations is shown by empty triangles (blue). Conjugate pairs of stations are PG5-SKT, PG4-UPN, PG3ATU, PG2-GDH, PG1-UMQ, PG0-KUV. Dotted lines show geographic coordinates, and solid lines denote corrected geomagnetic coordinates.

the main damping mechanisms is the ionospheric Joule dissipation (Newton et al., 1978). Another peculiarity of the resonant structure is the phase variation along latitude, whereas the largest gradient of the $B_{x}(x)$ phase is observed at the resonant shell. For typical magnetospheric conditions, the observed phase gradient corresponds to an apparent poleward propagation along a meridian.

\section{Comparison With Conjugate Observations of Pc5 Waves}

Here we compare the results of conjugate observations of Pc5 waves at high latitudes with theoretical predictions from Section 2.

\subsection{Database and Data Analysis Technique}

We use data from the conjugate Antarctica-Greenland magnetometer arrays. The Virginia Tech designed an autonomous adaptive low-power instrument platform (AAL-PIP). These instruments are deployed at sites in Antarctica that are magnetically conjugate to the Greenland West Coast magnetometer chain along the magnetic meridian $(L T \simeq U T-2.3)$. All stations are equipped with three-axis fluxgate magnetometers 
Table 1

Geographic and Geomagnetic Locations of the Greenland Stations and Conjugate Points of Antarctic Stations Based on the IGRF for Epoch 2015 (https://omniweb.gsfc.nasa.gov/vitmo/cgm.html)

\begin{tabular}{|c|c|c|c|c|c|}
\hline \multirow[b]{2}{*}{ AntarcticaGreenland } & \multirow[b]{2}{*}{ Code } & \multicolumn{2}{|c|}{ Geographic } & \multicolumn{2}{|c|}{ Geomagnetic conjugate } \\
\hline & & Latitude & Longitude & Latitude & Longitude \\
\hline & PG0 & -83.67 & 88.68 & 78.7 & 38.2 \\
\hline & PG1 & -84.50 & 77.20 & 77.3 & 37.3 \\
\hline & PG2 & -84.42 & 57.95 & 75.5 & 39.1 \\
\hline & PG3 & -84.81 & 37.63 & 73.8 & 36.6 \\
\hline & PG4 & -83.34 & 12.25 & 71.1 & 36.1 \\
\hline & PG5 & -81.96 & 05.71 & 69.7 & 37.0 \\
\hline Thule & THL & 77.47 & 290.77 & 84.4 & 27.5 \\
\hline Savissivik & SVS & 76.02 & 294.90 & 82.7 & 31.2 \\
\hline Kullorsuaq & KUV & 74.57 & 302.82 & 80.4 & 40.3 \\
\hline Upernavik & UPN & 72.78 & 303.85 & 78.6 & 38.7 \\
\hline Umanaq & UMQ & 70.68 & 307.87 & 76.0 & 41.2 \\
\hline Godhavn & GDH & 69.25 & 306.47 & 74.8 & 38.2 \\
\hline Attu & ATU & 67.93 & 306.43 & 73.5 & 37.1 \\
\hline Sondre Stromfjord & STF & 67.02 & 309.28 & 72.1 & 40.0 \\
\hline Sukkertoppen & SKT & 65.42 & 307.10 & 70.9 & 36.4 \\
\hline Godthaab & GHB & 64.17 & 308.27 & 69.5 & 37.1 \\
\hline Frederikshaab & FHB & 62.00 & 310.32 & 66.9 & 38.4 \\
\hline
\end{tabular}

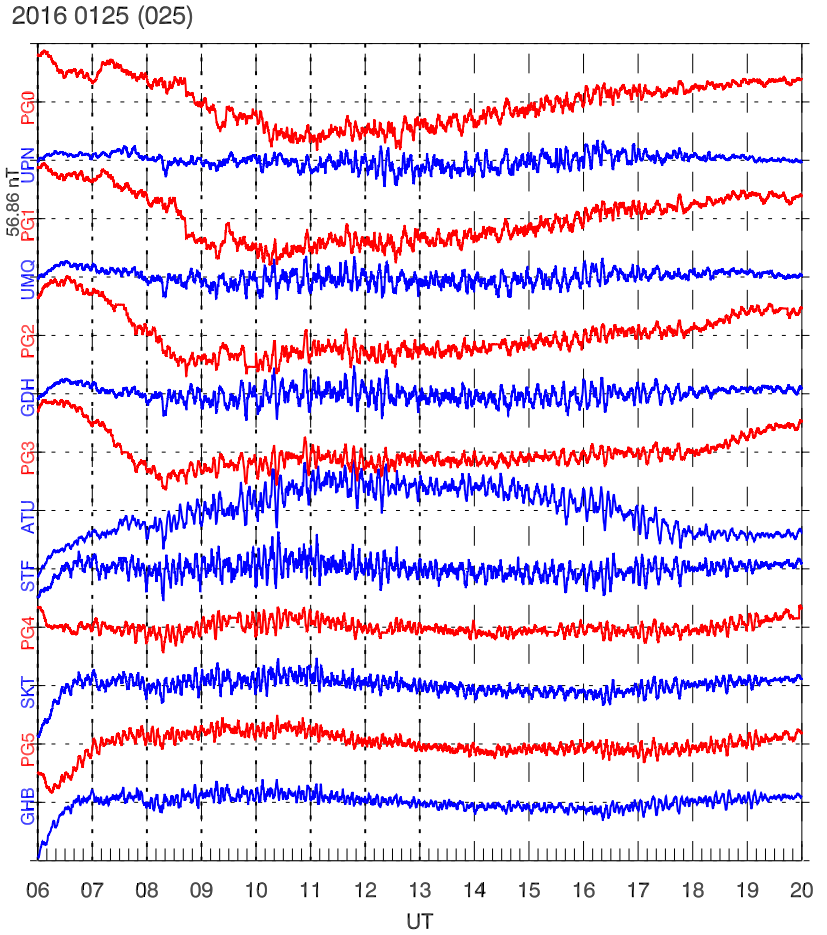

Figure 3. Stacked X-component magnetograms of quasi-narrowband Pc5 pulsations during Northern winter event of 2016, January 25 (year day 025), 06-20 UT along latitudinal profiles in Greenland (blue lines) and Antarctica (red lines). The scale between ticks is $56 \mathrm{nT}$. with 1-s cadence. The sensor axes are oriented along local magnetic north $\left(B_{x}\right)$, east $\left(B_{y}\right)$ and vertical down $\left(B_{z}\right)$. Figure 2 shows the locations of the Antarctic stations (in red color) and the geomagnetic conjugate points of their counterparts in Greenland (in blue color): PG0-UPN, PG1-UMQ, PG2-GDH, PG3-ATU, PG4-SKT, and PG5-GHB. Conjugate pair geocentric and geomagnetic coordinates are given in Table 1.

The difference in ionospheric conductances in opposite hemispheres is controlled by the solar illumination. This effect is accounted for by ionospheric models, for example, the Ionospheric Reference Ionosphere (IRI) (http://irimodel.org). Besides that, auroral electron precipitation influences the ionospheric conductivity. This factor is important for the considered problem, because the "epicenters" of the Pc5 power may be located near the equatorward oval boundary (Kozyreva et al., 2016). A source of information on the location of the auroral oval is the OVATION-prime (OP) model driven by solar wind and interplanetary magnetic field (IMF) parameters (http://sourceforge.net/projects/ovation-prime/). The OP model is based on energetic particle measurements from the Defense Meteorological Satellite Program (DMSP) satellites and considers the main types of aurorae: discrete monoenergetic and broadband diffuse (Newell et al., 2009). Diffuse aurora is caused by the precipitation of energetic electrons (0.1-30 keV) over a broad latitude range, and provides the main energy source for the high-latitude upper atmosphere (Newell et al., 2009). The discrete aurora at somewhat higher altitudes is dominated by soft electron precipitation (0.1-1 keV), which produce small additional ionization in the lower ionosphere. 
20160125 (025) 08:00-13:00

$\operatorname{fr}[\mathrm{mHz}]:\{2.5,3.0\}$
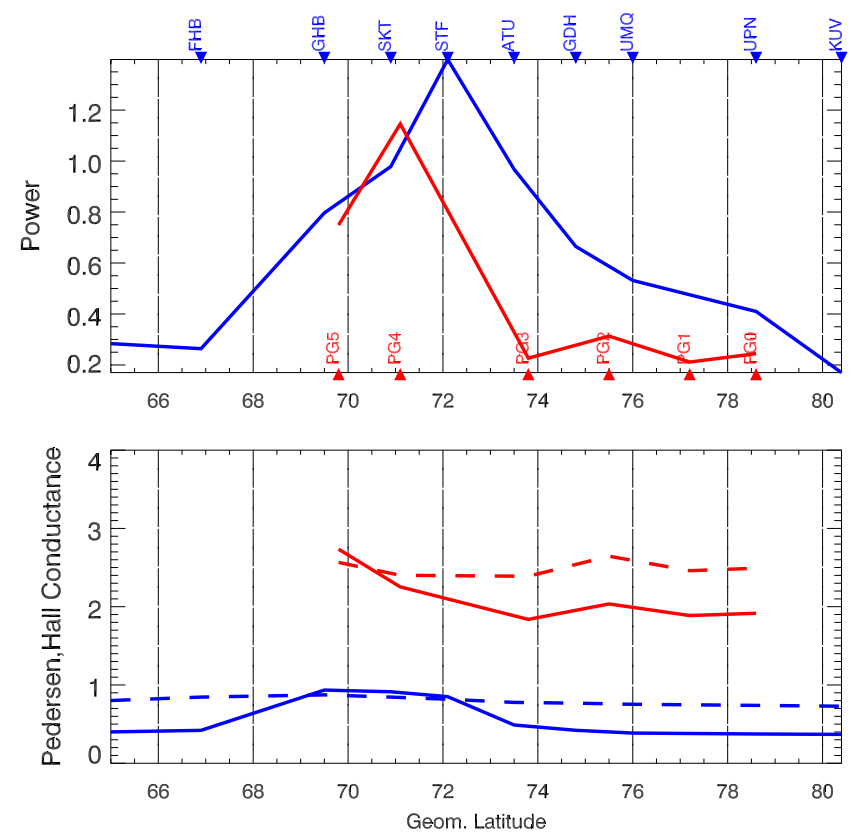

Figure 4. Latitudinal profiles for the Northern (blue lines) and Southern (red lines) hemispheres during the time interval 08-13 UT, January 25, 2016: (upper panel) the spectral amplitude density $\left[\right.$ in $\left.\mathrm{nT} / \mathrm{Hz}^{1 / 2}\right]$ in the frequency band $2.5-3.0 \mathrm{mHz}$ of the $B_{x}$ component; (bottom panel) the Pedersen (solid line) and Hall (dashed line) ionospheric conductances. Conductances were estimated with the use of the OP model. The position of stations in magnetic latitude is marked by filled triangles.
The height-integrated conductivities of conjugate ionospheres are estimated using the ionospheric parameters provided by the IRI-2016 model for a given time/location (http://wdc.kugi.kyoto-u.ac.jp/ionocond/ sightcal/). Besides that, we estimate the conductivity of the ionosphere taking into account contributions from both solar photoionization and auroral electron precipitation. As an estimator for electron precipitation contribution to ionospheric conductivity the method utilized in Cousins et al. (2015) has been used. The conductance as a result of photoionization is parameterized by F10.7 solar radio flux index and solar zenith angle at a particular location. The auroral average energy and energy flux contribution is modeled by the OP model and then related to Hall and Pedersen conductance by the formula proposed by Robinson et al. (1987). These two conductance products are then combined into a final estimate and averaged over the period of spectral analysis window.

A specific difficulty in analysis of interhemispheric properties of Pc5 pulsations is that resonant frequency-dependent amplification occurs in a small latitudinal region $\left(\delta_{g} \sim 200-300 \mathrm{~km}\right)$ and varying position of the resonant latitudinal maximum $x_{A}(f)$ from event to event. The conjugate observation results are expected to be strongly influenced by uncertainties in the difference between the pulsation resonant peak and an observation site. Therefore, any conclusions on Pc5 asymmetry demand a preliminary determination of spectral-latitudinal wave structure in both hemispheres.

The procedure of magnetometer data analysis includes the following steps. From visual inspection of magnetograms, the time intervals with quasi-monochromatic and stable Pc5 waves have been selected. The visual comparison of Pc5 wave amplitudes at different latitudes may be misleading, because the spectral content of pulsations varies with latitude owing to resonant effects. Indeed, notice a tendency of Pc5 pulsations to become more high frequency upon decrease of latitude (Figures 3 and 7).

Therefore, the analysis of spectral power at a selected frequency is more correct, but the location of the maximum of Pc5 spectral amplitude may depend on the selected frequency band. The data from all stations have been high-pass filtered with cut-off frequency $1 \mathrm{mHz}$, and spectral analysis has been performed in a time window with regular ULF signal. The spectrum was estimated with the standard Fast Fourier Transform periodogram method from the IDL tools. Latitudinal plots of $B_{x}(\Phi)$ spectral power at the central frequency of the selected band depending on geomagnetic latitude $\Phi$ were constructed. These plots enable us to compare the amplitudes of spatial peaks at two conjugate stations where the maxima are observed. At the same time, from the combined OP model the profiles of the ionospheric conductances are estimated. Using these values, we compare the observed ratios between Pc5 spectral power peaks and conductances in conjugate points with the theoretical predictions.

We have selected contrasting Pc5 events during the Northern summer and Northern winter. We suppose that most of Pc5 waves in our consideration are driven by the shear-flow instability, but with intermittent enhancements owing to the solar wind pressure jumps. For each event, stacked magnetograms of the $B_{x}$ component are presented from conjugate pairs of stations.

\subsection{Northern Winter Events}

For the January 25, 2016 event, the stacked raw magnetograms of $B_{x}$ component from conjugate pairs of stations are shown in Figure 3. Quasi-monochromatic Pc5 waves appear during 06-20 UT in the recovery phase of a weak substorm (magnetic bay $\sim 200 \mathrm{nT}$ ) with onset at $\sim 07$ UT. The most regular and monochromatic waves are observed at 08-17 UT. In the Northern hemisphere (Greenland) Pc5 pulsations are polarized in the N-S direction, that is $B_{x} \gg B_{y}$. At Antarctic stations the contrast between horizontal components is not so strongly pronounced, $B_{x} \quad B_{y}$. Signatures of field line resonance can be seen even from visual 
$20161112(317)$

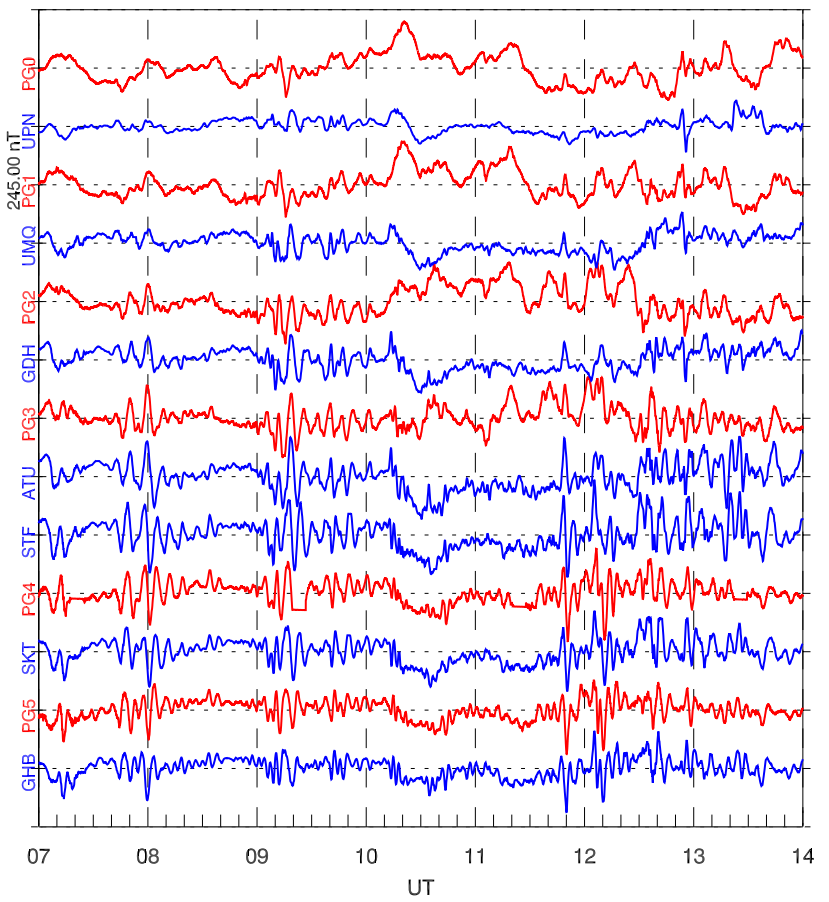

Figure 5. Stacked X-component magnetograms of narrowband Pc5 pulsations during Northern winter event of 2016, November 12 (year day 317), 07-14 UT along latitudinal profiles in Greenland (blue lines) and Antarctica (red lines). The scale between ticks is $196 \mathrm{nT}$. inspection of magnetograms in both hemispheres: a localized enhancement of magnetic variations around $\Phi \simeq 75^{\circ}$, apparent poleward phase propagation (evident after a zooming), dominance of the $B_{x}$ component over the $B_{y}$ component (not shown), and latitude-dependent frequency of Pc5 pulsations.

The spectra of $B_{x}$ component data (not shown) during the time interval 08-13 UT with stable Pc5 waves demonstrate the occurrence of a spectral peak at $2.5-3.0 \mathrm{mHz}$ at most stations in both Northern and Southern hemispheres. We examine the latitudinal structure of the spectral power of Pc5 waves in this narrow frequency band. The latitudinal distribution of spectral power along the profile has a maximum at $\Phi \simeq 71-72^{\circ}$ (STFPG4) in both hemispheres (Figure 4, upper panel). Spectral power in the Northern hemisphere is larger than that in the Southern hemisphere, but just slightly, by about $20 \%$.

For this event, the reconstructed ionospheric height-integrated conductances in the vicinity of the latitudinal peak of Pc5 spectral power are about $\Sigma_{H}^{(S)} \simeq 2.5 \mathrm{~S}, \Sigma_{P}^{(S)} \simeq 2.0 \mathrm{~S}$, and $\Sigma_{H}^{(N)} \simeq \Sigma_{P}^{(N)} \simeq 0.9 \mathrm{~S}$. According to the IRI model the contrast between conductances of Northern and Southern hemispheres at $10 \mathrm{UT}$ is to be much larger: $\Sigma_{H}^{(S)} \simeq 8.25 \mathrm{~S}, \Sigma_{P}^{(S)} \simeq 6.45 \mathrm{~S}$, and $\Sigma_{H}^{(N)} \simeq 0.17 \mathrm{~S}, \Sigma_{P}^{(N)} \simeq 0.14 \mathrm{~S}$. Therefore, the contrast between the Pc5 amplitudes, $\sim 20 \%$, is much less that the contrast between ionospheric conductances, $\sim 3.1$ according to the OP model, and $\sim 48$ according to the IRI model.

For the November 12, 2016 (day 317) event the stacked magnetograms of $B_{x}$ component data from conjugate pairs of stations for this day are shown in Figure 5. Several bursts of quasi-monochromatic Pc5 waves appeared during 07-14 UT. The most intense monochromatic waves we

re observed at time intervals 09-10 UT and 11-30 to 13-30 UT. The central frequency along the profile varied between 2.5 and $4.0 \mathrm{mHz}$ (spectra are not shown).

The latitudinal profiles along $\Lambda=40^{\circ}$ for the Northern (blue lines) and Southern (red lines) hemispheres during the time interval 11-30 to 14-00 UT, November 12, 2016 of the spectral power density (in the frequency band 2.5-3.0 mHz) of the $B_{x}$ component are shown in Figure 6. The bottom panel of this figure shows the latitudinal profile of the Pedersen (solid line) and Hall (dashed line) conductances reconstructed with the OP model. The ionospheric conductances vary smoothly with latitude indicating that the auroral oval is beyond the range of geomagnetic latitudes $70-78^{\circ}$ under detailed consideration. The peak of the latitudinal distribution of spectral power in both Northern and Southern hemispheres is around $\Phi \simeq 71-72^{\circ}$ (STFPG4). The ionospheric conductances in the Southern hemisphere, $\Sigma_{H}^{(S)} \sim 7.5 \mathrm{~S}$ and $\Sigma_{P}^{(S)} \sim 6.0 \mathrm{~S}$, are much larger than those in the Northern hemisphere, $\Sigma_{H}^{(S)} \simeq \Sigma_{H}^{(S)} \sim 0.9 \mathrm{~S}$, that is $\sim 8.3$ times. According to the IRI model the contrast between conductances of Northern and Southern hemispheres at 12 UT is even larger: $\Sigma_{H}^{(S)} \simeq 7.7 \mathrm{~S}, \Sigma_{P}^{(S)} \simeq 5.8 \mathrm{~S}$, and $\Sigma_{H}^{(N)} \simeq 0.6 \mathrm{~S}, \Sigma_{P}^{(N)} \simeq 0.5 \mathrm{~S}$, that is $\sim 12$ times. However, the spectral peaks of Pc5 pulsations in both hemispheres are nearly the same.

Therefore, the consideration of Northern winter events has shown that Pc5 wave excitation is much closer to the current generator regime than to the voltage regime.

\subsection{Northern Summer Events}

For the event of June 02, 2016 (day 154), the stacked raw magnetograms of $B_{x}$ component data from conjugate pairs of stations are shown in Figure 7. Pc5 waves appeared during 08-12 UT. The most intense 
$20161112(317)$ 11:30-14:00
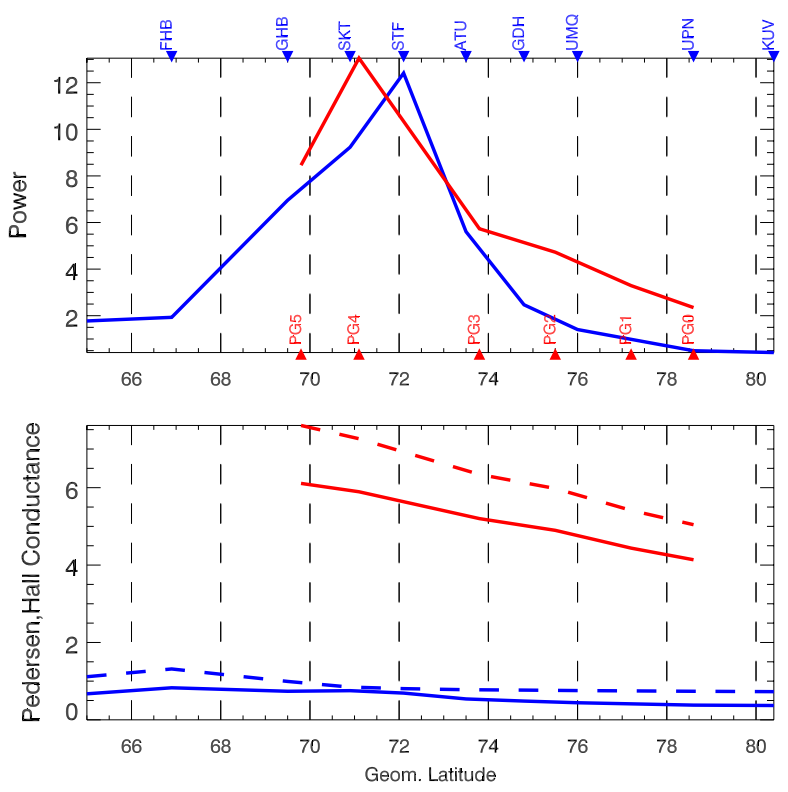

Figure 6. Latitudinal profiles for the Northern (blue lines) and Southern (red lines) hemispheres during the time interval 11-30 to 14-00 UT, November 12, 2016: (upper panel) of the spectral power density in the frequency band $2.5-3.0 \mathrm{mHz}$ of the $B_{x}$ component; (bottom panel) the Pedersen (solid line) and Hall (dashed line) ionospheric conductances. The position of stations in magnetic latitude is marked by filled triangles. monochromatic waves were observed at 09-10 UT. The central frequency along the profile varied between 3.0 and $4.0 \mathrm{mHz}$ (spectra are not shown).

The latitudinal profiles for the Northern (blue lines) and Southern (red lines) hemispheres during the time interval 08-30 to 10-30 UT of the spectral power density (in the frequency band $3.5-4.0 \mathrm{mHz}$ ) of the $B_{x}$ component are shown in Figure 8. The peaks of the latitudinal distribution of spectral power in the Northern and Southern hemispheres are at $\sim 74^{\circ}$ (ATU-PG3) and nearly of the same magnitude (difference is 25\%). The profile of the Pedersen (solid line) and Hall (dashed line) ionospheric conductances reconstructed with the OP model vary smoothly with latitude indicating that the oval is beyond the range of geomagnetic latitudes under detailed consideration or the auroral precipitation is weak.

The ionospheric conductances in the Northern hemisphere, $\Sigma_{H}^{(N)} \simeq 6$ $\mathrm{S}$ and $\Sigma_{P}^{(N)} \simeq 4.8 \mathrm{~S}$, are much larger than in the Southern hemisphere, $\Sigma_{H}^{(S)} \simeq \Sigma_{P}^{(S)} \sim 1 \mathrm{~S}$. Thus, the contrast between Pc5 spectral amplitudes between hemispheres, $\sim 25 \%$, is much less than the contrast between the ionospheric conductances, 5-6 times. According to the IRI model the contrast between the Hall conductances of Northern and Southern hemispheres at $09 \mathrm{UT}$ is even larger, $\sim 25$ times, $\Sigma_{H}^{(S)} \simeq 0.2 \mathrm{~S}$, and $\Sigma_{H}^{(N)} \simeq 5.1 \mathrm{~S}$.

For the event of June 13, 2016 (day 165), the stacked magnetograms of $B_{x}$ component data from conjugate pairs of stations are shown in Figure 9. Quasi-monochromatic Pc5 waves appeared during 09-15 UT, and the most intense monochromatic waves were observed at 11-14 UT. Even from visual inspection of magnetograms one can see a regular increase of dominant frequency from $2.5 \mathrm{mHz}$ at high latitudes (PG2-UMQ) toward $3.5 \mathrm{mHz}$ at lower latitudes (PG5-SKT).

During the 11-00 to 13-00 UT interval the latitudinal peaks of spectral power at $f=2.5-3.0 \mathrm{mHz}$ were at the same geomagnetic latitude in both hemispheres, around $74-74^{\circ}$ (GDH/PG3) (Figure 10). The contrast between ionospheric conductances is rather substantial, $\Sigma_{H}^{(N)} \simeq 10.5 \mathrm{~S}$, whereas $\Sigma_{H}^{(S)} \simeq 1.0 \mathrm{~S}$. The profile of the ionospheric conductance is weakly dependent on latitude, and strong gradients are absent. According to the IRI model, the contrast between the Hall conductances of Northern and Southern hemispheres at 12 UT is even larger, $\sim 36$ times, as follows $\Sigma_{H}^{(S)} \simeq 0.2 \mathrm{~S}$, and $\Sigma_{H}^{(N)} \simeq 7.2 \mathrm{~S}$. Despite the strong asymmetry of the ionospheric conductance, about an order of magnitude, the magnetic field spectral power was nearly the same in both hemispheres, the difference is $\sim 10 \%$.

\section{Discussion}

The solution of the problem of the excitation of FAC in the magnetospheric resonator shows that the fieldaligned structure and dependence on the ionospheric conductance may look like a voltage or current generator, depending on the periodicity of the driver regime $\tau$ and the local eigenperiod of the magnetospheric resonator $T_{A}$ (Pilipenko et al., 2019). For a quasi-DC forced driving $\left(\tau \gg T_{A}\right.$ ), the structure of the disturbance corresponds to the voltage regime, when the magnetic response is proportional to the ionospheric conductance. In the case of resonant driving $\left(\tau \simeq T_{A}\right)$, the structure of the excited disturbance corresponds to a current generator, when the magnetic response practically does not depend on the ionospheric conductance. The consideration of the current/voltage dichotomy for ULF waves (Pc3-5 pulsations) should be done with great care. The ULF wavefield is composed from the fast magnetosonic (compressional) mode and the Alfven mode, because the wave energy transport from the outer into the inner magnetosphere is performed by coupled MHD modes. The transmission mechanisms of the compressional mode and Alfven mode through the ionosphere are very different (Vorontsova et al., 2016). While a FAC carried by an Alfven wave cannot penetrate into the insulating atmosphere and spreads over the ionosphere as Pedersen and Hall currents, the magnetic field compression transported by a fast mode wave "feels" the ionosphere only 
20160602 (154)

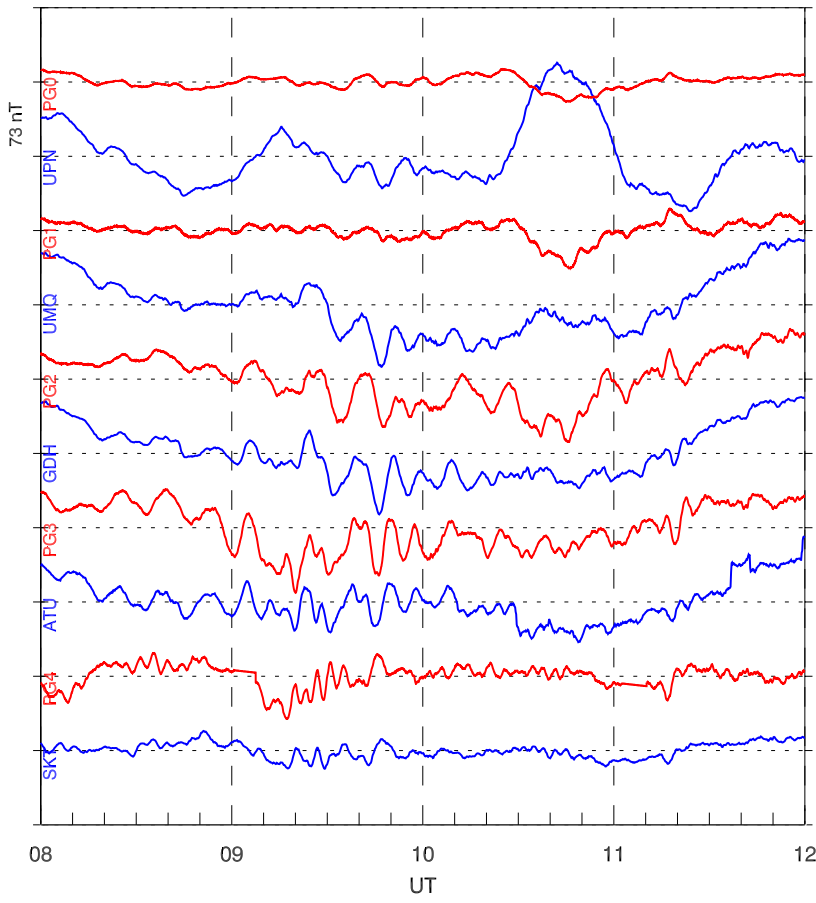

Figure 7. Stacked X-component magnetograms of narrowband Pc5 pulsations during the Northern summer event of 2016, June 02, 08-12 UT along latitudinal profiles in Greenland (blue lines) and Antarctica (red lines). The scale in nT between ticks is shown near left-hand $X$-axis.

\section{2 (154) 08:30-10:30}
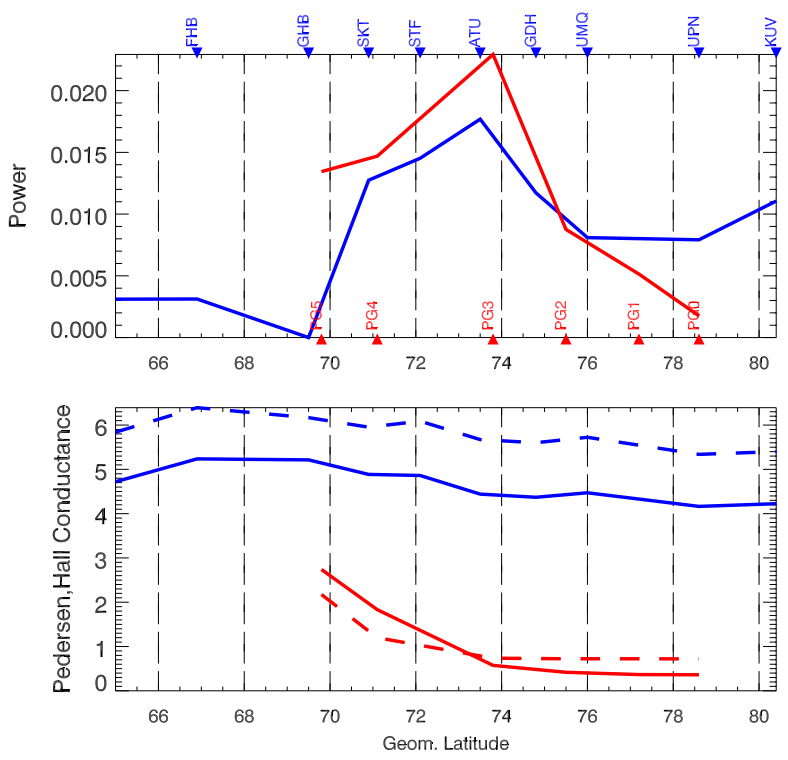

Figure 8. Latitudinal profiles for the Northern (blue lines) and Southern (red lines) hemispheres during the time interval 08-30 to 10-30 UT, June 02 (year day 154), 2016: (upper panel) of the spectral power density (in the frequency band $3.5-4.0 \mathrm{mHz}$ ) of the $B_{x}$ component; (bottom panel) of the Pedersen (solid line) and Hall (dashed line) ionospheric conductances. The position of stations in magnetic latitude is marked by filled triangles. weakly. Both modes, when having large azimuthal spatial scales, produce a main ground response in the same North-South component. Therefore, for the examination of conjugacy features, one has to use the wave spectral amplitudes in the vicinity of the resonant maxima where the contribution of the Alfvenic mode dominates. The interpretation scheme used here cannot be applied to Pi2 pulsations because these waves are mainly composed of the fast compressional mode (Takahashi et al., 1995).

The current/voltage generator paradigm was regularly tested with observations at conjugate points (Saito et al., 1989). The north-south asymmetry of the amplitude of Pc5 pulsations was studied by Obana et al. (2005) using magnetic field data from the conjugate Kotzebue-Macquarie Island stations at $L \simeq 5.4$. The power ratio showed a "regular offset," probably caused by a regular shift of the statistical position of the wave resonant peak and the station location. The relative stability of the offset during seasonal variations may indirectly be evidence of the independence of the magnetic signal on the ionospheric conductance, and thus be evidence of the Pc5 wave driver as a current generator.

Our consideration has given evidence that amplitudes of resonant Pc5 pulsation peaks are about the same during both Northern winter and summer, and the difference typically does not exceed $20 \%$. Our comparison of spectral power latitudinal profiles in Antarctica and Greenland has shown that the forms of profiles in both hemispheres may be different. In these cases, the comparison of spectral power at selected stations even at the same $\Phi$ may provide a substantial difference in power at conjugate stations. That is why we suppose that interhemispheric differences in Pc5 pulsations should be examined with latitudinal arrays only, but not isolated conjugate stations. For illustration, let us consider the June 13, 2016 event. While the spectral peak difference is only $20 \%$, the ratio between Pc5 spectral amplitudes between conjugate stations away from the resonant peak can be $\sim 3$ times (cf. UMQ-PG2 pair at $\Phi=76^{\circ}$ ).

Typically, maxima of Pc5 amplitudes are observed in Antarctica at stations PG4-PG3. Their geomagnetic latitudes, $\sim 71-73^{\circ}$, do not characterize fully the magnetospheric field line configuration because standard CGM coordinates are based on internal magnetic field sources only. The mapping of field lines corresponding to these stations into the magnetosphere with the help of the Tsyganenko-2001 model (https://ccmc.gsfc. nasa.gov/requests/instant/tsyganenko.php) provides the distance to the magnetic field line apex about $10-12 R_{E}$ depending on LT. This modeling shows that though the magnetic field line is distorted as compared with a dipole model (e.g., the minimum of $B_{0} \sim 15-40 \mathrm{nT}$ is shifted away from the $Z=0$ plane and the field lines are somewhat dragged tailward), the field lines are always closed. The modeling for other Pc5 events provides nearly the same estimates, the modeled parameters differ not more than $15 \%$. This is consistent with past work that has also identified FLR at similar latitudes (Mathie \& Mann, 2000).

So far, no check of a match between the observed Pc5 central frequency and modeled eigenfrequency can be done reliably. Calculations of the Alfven eigenfrequency of the field line demand realistic magnetic field and magnetospheric plasma models. While more or less realistic magnetic field models exist, there is no information on the field-aligned plasma distribution and its ion content $\left(\mathrm{H}^{+}, \mathrm{O}^{+}, \mathrm{He}^{+}\right)$. Without this information, any calculations of resonant frequency would be valid only within an or- 
$20160613(165)$

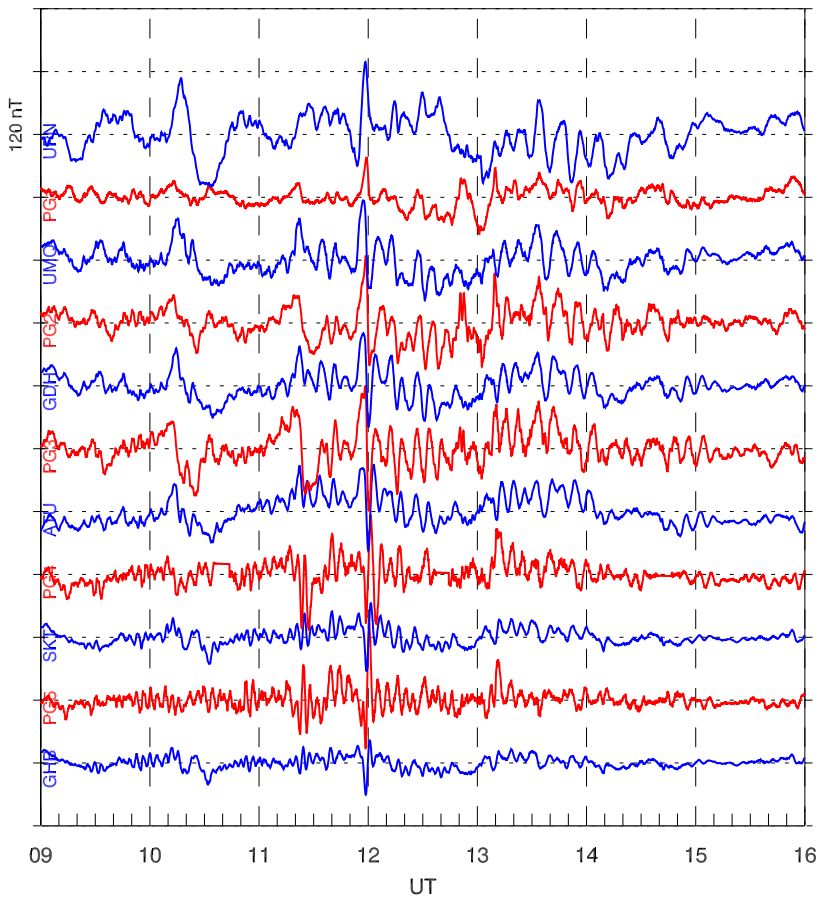

Figure 9. Stacked $X$-component magnetograms of narrowband Pc5 pulsations during the Northern summer event of 2016, June 13 (year day 165), 09-16 UT along longitudinal profiles in Greenland (blue lines) and Antarctica (red lines). The scale in nT between ticks is shown near lefthand $X$-axis. der of magnitude. We can at least say that $2.5-3.0 \mathrm{mHz}$ is consistent with expectations based on past predictions for realistic magnetic field geometries. Singer et al. (1981) assumed an equatorial plasma density of $1 \mathrm{~cm}^{-3}$ in a distorted dipole, and found that the frequency in the $71-72^{\circ}$ range was on the order of $3 \mathrm{mHz}$ prenoon, depending strongly on LT, though this estimate will change as the assumed density changes.

In addition, in most magnetospheric MHD wave models, the magnetospheric plasma density and magnetic field are azimuthally homogeneous. Therefore, the Alfven shell $T_{A}(r)$ (location of equal eigenperiods of Alfven oscillations) coincides with a geomagnetic shell, characterized by the geomagnetic latitude $\Phi$. In general, the magnetospheric field lines at high latitudes are not confined by the meridional plane, but are dragged tailward, and plasma density is azimuthally inhomogeneous. The resonance theory for such a case can be constructed only numerically (Lee et al., 2000). As a result, on the ground the $T_{A}(r)$ location may be different from the $B_{0}(r)$ location. Therefore, the conjugacy of magnetospheric field lines may not coincide with the conjugacy of resonant ULF oscillations. This feature may be a reason that the spectral peaks in opposite hemispheres sometimes are observed at slightly different latitudes (the separation $\sim 1^{\circ}$ ), e.g., the event on January 25, 2016 (Figure 4).

The key problem of interhemispheric studies is the availability of information on the actual ionospheric conductances at conjugate points. The IRI model does not incorporate the effect of energetic electron precipitation and predicts a very large difference between conductances in opposite hemispheres. The precipitation at high latitudes may noticeably smooth the interhemispheric difference between the ionospheric conductance parameters $\Sigma_{H}^{(N)} / \Sigma_{H}^{(S)}$ and $T^{(N, S)}$ (Hartinger et al., 2017). The precipitation effect may significantly influence the conjugacy properties of Pc5 waves, because the epicenter of their activity is close to the auroral oval. In our analysis we lack riometer or auroral data, but we used the realistic OP model for selected events with latitudinal maxima away from the strong gradient of ionospheric conductance. A steep gradient of the ionospheric conductance, e.g., at the auroral oval boundary or cusp boundary, can substantially distort a ground magnetic response (Glassmeier, 1984).

Though the model considered here is homogeneous, in reality the plasma distribution along a field line must be inhomogeneous: the plasma density $N_{e}$ in the upper ionosphere at the sunlit end of a field line should be higher than at the dark end. As a result, the Alfven wave conductance, $\Sigma_{A} \propto \sqrt{N_{e}}$, must be higher at the sunlit end. Thus, the contrast in the ratio $\Sigma_{P} / \Sigma_{A}$, which determines the reflection condition and ground response, is expected to be less distinct between Northern and Southern ionospheres. As a proxy of $N_{e}$ in the upper ionosphere TEC maps can be used. Nonetheless, the expected correction factor would hardly exceed a few tens of percent (Pilipenko et al., 2020). In a realistic magnetosphere-ionosphere system, the plasma distribution along a field line is inhomogeneous, so it can be characterized by a single value such as $\Sigma_{A}$ only qualitatively, but demands numerical calculations of field-aligned eigenfunction. Finally, some offset between ULF wave amplitudes in opposite hemispheres may be produced by different geoelectric properties of the crust under the conjugate stations.

The observed independence of the interhemispheric ratio of Pc5 spectral power on the local ionospheric conductance just indicates that the wave excitation regime operates as a current generator. However, this does not mean that the Pc5 pulsation excitation rate is not influenced by the ionospheric conductivity. On the contrary, there is evidence that such ionospheric domains with enhanced ionospheric conductivity as the auroral oval and westward auroral electrojet are a preferred region for Pc5 wave excitation (Sutcliffe \& Rostoker, 1979). However, there is not an adequate theoretical interpretation for this feature yet. 

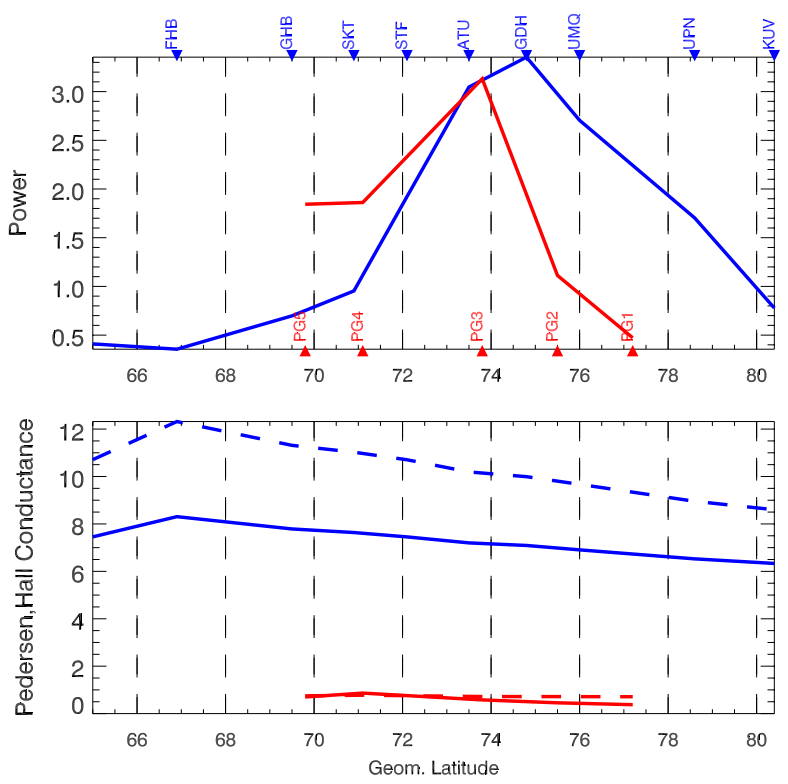

Figure 10. Latitudinal profiles for the Northern (blue lines) and Southern (red lines) hemispheres during the time interval 11-00 to 13-00 UT, June 13, 2016: (upper panel) of the spectral power density in the frequency band 2.5-3.0 mHz of the $B_{x}$ component; (bottom panel) of the Pedersen (solid line) and Hall (dashed line) ionospheric conductances. The position of stations in magnetic latitude is marked by filled triangles.
There is still ongoing search for signatures of a quarter-wave mode (the most recent study in this direction is by Obana et al. (2008)). This mode is expected to have an anomalously long period as compared with Pc5 pulsations at the same latitude. Occurrence of a quarter-wave mode is to be a rather rare phenomenon because a high interhemispheric contrast should exist between normalized conductances $\bar{\Sigma}_{P}$, while both $\Sigma_{P}$ and $\Sigma_{A}$ depend on the ionosphere-plasmasphere density in a similar way. The relation (4) predicts a strong asymmetry between geomagnetic responses at conjugate sites which may be used as an additional signature of a quarter-wave mode. This relationship also indicates the occurrence of an additional phase shift $\sim \pi / 2$ between pulsations at conjugate sites.

\section{Conclusion}

According to the suggested theoretical conception such magnetospheric phenomena as Pc5 waves should be considered as a resonant response of magnetospheric field lines and they correspond to a current generator. We have analyzed the asymmetry of Pc5 waves recorded at conjugate magnetometer arrays in Greenland and Antarctica from the viewpoint of the voltage/current generator dichotomy. The analysis of Pc5 events under contrasting ionospheric conditions during the summer and winter has shown that amplitudes of the spectral power peaks at the resonant frequency in Greenland and Antarctica are about the same (may differ not more than by $20 \%$ ), though the ionospheric conductance model predicts a contrast between ionospheric conductivities of 3-10 times. Thus, the conjugacy properties of Pc5 waves correspond to the current generator regime, rather than to the voltage generator.

This work, combined with past results in Pilipenko et al. (2019), indicates that the temporal and spatial scale of magnetospheric driving conditions and proximity to resonant field lines all play significant roles in determining the ground magnetic response for a given ionospheric conductivity profile. This conclusion has important implications for global simulations seeking to predict ground magnetic perturbations: though the ionospheric conductivity is an important parameter needed to predict ground magnetic perturbations, other parameters (driver time scale, local Alfven eigenperiod) can play equally important roles.

\section{Data Availability Statement}

The OVATION-Prime model was developed at Johns Hopkins Applied Physics Laboratory by P. Newell and coworkers (http://sd-www.jhuapl.edu/Aurora). The Antarctic magnetometer data are freely available from the home page for the Virginia Tech AAL-PIP project (http://mist.nianet.org). DTU data from Greenland are obtained from the public Tromso Geophysical Observatory website (http://flux.phys.uit.no/geomag. $\mathrm{html}$ ). The useful suggestions of the reviewers are appreciated.

\section{Acknowledgments}

This study is supported by the Russian Fund for Basic Research Grant 20-0500787 (Valery A. Martines-Bedenko and Evgeny N. Fedorov), and U.S. National Science Foundation Grants PLR-1744828 to Virginia Tech (Michael D. Hartinger and Shane Coyle) and PLR-1341493 and AGS-1639587 to Augsburg University (Vyacheslav A. Pilipenko and Mark J. Engebretson).

\section{References}

Alperovich, L. S. \& Fedorov, E. N. (2007). Hydromagnetic waves in the magnetosphere and the ionosphere, Astrophysics and Space Science Library (Vol.353, XXIV, pp. 418). Netherland: Springer.

Chen, L., \& Hasegawa, A. (1974). A theory of long-period magnetic pulsations. 1: Steady state excitation of field line resonance. Journal Geophysical Research, 79, 1024-1032.

Cousins, E. D. P., Matsuo, T., \& Richmond, A. D. (2015). Mapping high-latitude ionospheric electrodynamics with SuperDARN and AMPERE. Journal Geophysical Research: Space Physics, 120, 5854-5870. https://doi.org/10.1002/2014JA020463

Glassmeier, K.-H. (1984). On the influence of ionospheres with non-uniform conductivity distribution on hydromagnetic waves. Journal Geophysical Research, 54, 125-137.

Hartinger, M. D., Xu, Z., Clauer, C. R., Yu, Y., Weimer, D. R., Kim, H., et al. (2017). Associating ground magnetometer observations with current or voltage generators. Journal Geophysical Research: Space Physics, 122, 7130-7141. https://doi.org/10.1002/2017JA024140

Hughes, W. J., \& Southwood, D. J. (1976). The screening of micropulsation signals by the atmosphere and ionosphere. Journal Geophysical Research, 81, 3234-3240. 
Kim, H., Clauer, C. R., Engebretson, M. J., Matzka, J., Sibeck, D. G., Singer, H. J., et al. (2015). Conjugate observations of traveling convection vortices associated with transient events at the magnetopause. Journal Geophysical Research: Space Physics, 120, 2015-2035. https:// doi.org/10.1002/2014JA020743

Kivelson, M. G., \& Southwood, D. J. (1986). Coupling of global magnetospheric MHD eigenmodes to field line resonances. Journal Geophysical Research, 91, 4345-4351.

Kozyreva, O. V., Pilipenko, V. A., Engebretson, M. J., Klimushkin, D. Y., \& Mager, P. N. (2016). Correspondence between the ULF wave power distribution and auroral oval. Solar-Terrestrial Physics, 2, 46-65. https://doi.org/10.12737/20999

Krylov, A. L., Lifshitz, A. E., \& Fedorov, E. N. (1979). About resonant properties of a plasma in a curvilinear magnetic field. Doklady AN SSSR, 247, 1095-1096.

Krylov, A. L., Lifshitz, A. E., \& Fedorov, E. N. (1980). About resonant properties of the magnetospheric field lines. Geomagnetism Aerono$m y, 20,689-692$.

Lam, M. M., \& Rodger, A. S. (2004). A test of the magnetospheric source of traveling convection vortices. Journal Geophysical Research, 109, A02204. https://doi.org/10.1029/2003JA010214

Lee, D.-H., Lysak, R. L., \& Song, Y. (2000). Field line resonances in a nonaxisymmetric magnetic field. Journal Geophysical Research, 105 10703-10712.

Lysak, R. L. (1985). Auroral electrodynamics with current and voltage generators. Journal Geophysical Research, 90, 4178-4190.

Lysak, R. L. (1990). Electrodynamic coupling of the magnetosphere and ionosphere. Space Science Reviews, 52, 33-87.

Mathie, R. A., \& Mann, I. R. (2000). Observations of Pc5 field line resonance azimuthal phase speeds: A diagnostic of their excitation mechanism. Journal Geophysical Research, 105, 10713-10728. https://doi.org/10.1029/1999JA000174

Menk, F. W., \& Waters, C. L. (2013). Magnetoseismology: Ground-based remote sensing of earth's magnetosphere. Weinheim, Germany:Wiley-VCH Verlag GmbH \& Co. https://doi.org/10.1002/9783527652051

Mishin, V. V. (1993). Accelerated motions of the magnetopause as a trigger of the Kelvin-Helmholtz instability. Journal Geophysical Research, 98, 21365-21371.

Newell, P. T., Sotirelis, T., \& Wing, S. (2009). Diffuse, monoenergetic, and broadband aurora: The global precipitation budget. Journal Geophysical Research, 114, A09207. https://doi.org/10.1029/2009JA014326

Newton, R. S., Southwood, D. J., \& Hughes, W. J. (1978). Damping of geomagnetic pulsations by the ionosphere. Planetary Space Science, 26, 201-209.

Obana, Y., Menk, F. W., Sciffer, M. D., \& Waters, C. L. (2008). Quarter-wave modes of standing Alfven waves detected by cross-phase analysis. Journal Geophysical Research, 113, A08203. https://doi.org/10.1029/2007JA012917

Obana, Y., Yoshikawa, A., Olson, J. V., Morris, R. J., Fraser, B. J., \& Yumoto, K. (2005). North-south asymmetry of the amplitude of high-latitude Pc3-5 pulsations: Observations at conjugate stations. Journal Geophysical Research, 110, A10214. https://doi. org/10.1029/2003JA010242

Pilipenko, V., Kozyreva, O., Fedorov, E., Uspensky, M., \& Kauristie, K. (2016). Latitudinal amplitude-phase structure of MHD waves: STARE radar and IMAGE magnetometer observations and modeling. Solar-Terrestrial Physics, 2(N3), 41-51. https://doi.org/10.12737/19418

Pilipenko, V., Fedorov, E., Xu, Z., Hartinger, M. D., Engebretson, M. J., \& Edwards, T. R. (2020). Incidence of Alfvenic SC pulse onto the conjugate ionospheres. Journal Geophysical Research: Space Physics, 125 e2019JA027397. https://doi.org/10.1029/2019JA027397

Pilipenko, V. A., Fedorov, E. N., Hartinger, M. D., \& Engebretson, M. J. (2019). Electromagnetic fields of magnetospheric ULF disturbances in the ionosphere: Current/voltage dichotomy. Journal Geophysical Research: Space Physics, 124, 109-121. https://doi. org/10.1029/2018JA026030

Robinson, R. M., Vondrak, R. R., Miller, K., Dabbs, T., \& Hardy, D. (1987). On calculating ionospheric conductances from the flux and energy of precipitating electrons. Journal Geophysical Research, 92, 2565-2569. https://doi.org/10.1029/ja092ia03p02565

Saito, H., Sato, N., Tonegawa, Y., Yoshino, T., \& Saemundsson, T. (1989). Seasonal and diurnal dependence of Pc3-5 magnetic pulsation power at geomagnetically conjugate stations in the auroral zones. Journal Geophysical Research, 94, 6945-6948.

Sibeck, D. G., Greenwald, R. A., Bristow, W. A., \& Korotova, G. I. (1996). Concerning possible effects of ionospheric conductivity upon the occurrence patterns of impulsive events in high-latitude ground magnetograms. Journal Geophysical Research, 101, 13407-13412.

Singer, H. J., Southwood, D. J., Walker, R. J., \& Kivelson, M. G. (1981). Alfven wave resonances in a realistic magnetospheric magnetic field geometry. Journal Geophysical Research, 86, 4589-4596.

Southwood, D. J. (1974). Some features of field line resonances in the magnetosphere. Planetary Space Science, 22, $483-491$.

Sutcliffe, P. R., \& Rostoker, G. (1979). Dependence of Pc5 micropulsation power on conductivity variations in the morning sector. Planetary Space Science, 27, 631-642.

Takahashi, K., Ohtani, S., \& Anderson, B. J. (1995). Statistical analysis of Pi2 pulsations observed by the AMPTE CCE spacecraft in the inner magnetosphere. Journal Geophysical Research, 100, 21929-21941.

Vorontsova, E., Pilipenko, V., Fedorov, E., Sinha, A. K., \& Vichare, G. (2016). Modulation of total electron content by global Pc5 waves at low latitudes. Advances in Space Research, 57, 309-319. https://doi.org/10.1016/j.asr.2015.10.041

Yumoto, K., Pilipenko, V., Fedorov, E., Kurneva, N., \& Shiokawa, K. (1995). The mechanisms of damping of geomagnetic pulsations. Journal Geomagnetism Geoelectricity, 47, 163-176.

Zesta, E., Boudouridis, A., Weygand, J. M., Yizengaw, E., Moldwin, M. B., \& Chi, P. (2016). Inter-hemispheric asymmetries in magnetospheric energy input. In T. Fuller-Rowell, E. Yizengaw, P. H. Doherty, \& S. Basu (Eds.), Ionospheric space weather: longitude dependence and lower atmosphere forcing, Geophysical Monograph Series (Vol. 220, pp. 1). Washington, DC: American Geophysical Union. https:// doi.org/10.1002/9781118929216.ch1 